\title{
Has the short-term effect of black smoke exposure on pneumonia mortality been underestimated because hospitalisation is ignored: findings from a case-crossover study
}

Matthew Gittins ${ }^{1 *}$, Roseanne McNamee ${ }^{1}$, Melanie Carder $^{2}$, lain Beverland ${ }^{3}$ and Raymond M Agius ${ }^{2}$

\begin{abstract}
Background: Short-term associations have been demonstrated between air pollution and respiratory mortality including pneumonia. Studies typically estimate exposure based only on place of residence, yet many are in hospital prior to death. This study investigates lag length and tests the hypothesis that the effect of 'black smoke' is greater when restricted to pneumonia deaths in the community - Community Deaths from Pneumonia.

Methods: A time-stratified case-crossover design using conditional logistic regression estimated the daily percentage increase in risk of pneumonia mortality in relation to 'black smoke' in the preceding 30 days. Cases were pneumonia deaths in Edinburgh 1981-1996. Multiple 'control' periods, were defined using the same weekdays for the same month as the case death. Lag structure was investigated by a stratified lag model with five 6-day periods and by distributed lag models. Hospital admissions data, defined a community death as someone who had not been in hospital in any of the 30 days before death.
\end{abstract}

Results: Of 14,346 subjects who died from pneumonia, 7,536 were community deaths. Larger estimated increases in risks were seen in the community for all lag periods. Both stratified and distributed lag methods suggested positive effect estimates for 18 days after exposure and negative thereafter; the average percent increase per day across the 18 days was $0.70 \%$ (95\% C.I. 0.29-1.14) for community subjects and $0.30 \%$ (95\% C.I. 0.03-0.59) for all subjects.

Conclusions: Studies which fail to account for hospitalisation may underestimate exposure effects as stronger pollution effects on mortality were evident in community based subjects.

Keywords: Air pollution, Time-stratified, Case-crossover, Community, Lag stratified, Lag distributed

\section{Background}

Many studies worldwide have demonstrated an association between air pollution and all cause mortality $[1,2]$, specifically respiratory mortality [3-5]. In 2011, pneumonia was the $6^{\text {th }}$ leading cause of death in England and Wales for males $(10,824)$ and $4^{\text {th }}(14,872)$ for females [6]. However, only a few studies - with limited findings - have specifically investigated associations between pneumonia related deaths and ambient air pollution. Schwartz and

\footnotetext{
* Correspondence: Matthew.Gittins@manchester.ac.uk

'Biostatistics Group, University of Manchester, Jean McFarlane Building, Oxford Road, Manchester M13 9PL, England, UK

Full list of author information is available at the end of the article
}

Dockery, indicated an increase in pneumonia mortality of

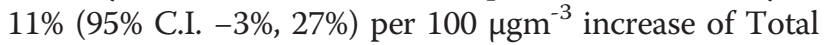
Suspended Particles (TSP) [7]. Halonen et al. demonstrated a percentage increase in pneumonia mortality in Finland of $3.16 \%$ (95\% C.I. $-2.64 \%, 9.32 \%$ ) per increase in inter-quartile range of a 5 day Coarse Particle Matter $\left(\mathrm{PM}_{10-2.5}\right)$ mean [8]. Zanobetti et al. proposed that air pollution may be a predisposing factor to Community Acquired Pneumonia (CAP) and that subjects with CAP rather than Hospital Acquired Pneumonia, may be more susceptible to the effects of air pollution [9]. Studies such as Neupane et al. have indicated a relationship between long-term exposure to air pollution and emergency

\section{Biomed Central}


visits to hospital with Community Acquired Pneumonia [10]. However, so far no study has investigated the effect of pollution on deaths from Community Acquired Pneumonia only.

Much of the evidence for the association between air pollution and general mortality has focused on exposure in a short time period - less than 40 days prior to death $[2,11]$. This focus on short to medium exposure may be appropriate for pneumonia which is generally an acute condition, though often associated with chronic underlying lung disease. Frequently, a deceased individual's exposure is inferred from information regarding their place of residence with little or no attempt to take account of subject's actual location, circumstances or activities. Exposure is typically assumed to be the same for all subjects living within a given distance from a single pollution monitor or an average of multiple monitors within the area $[1,11]$. More recent studies, trying to improve exposure estimates, have taken into account traffic density and other geographical information regarding the subjects neighbourhood or city $[12,13]$. The presumption still remained that the deceased was in the geographical location of residence during the exposure period; in fact it is common for people to die in non-residential locations (65.3\% in a NHS hospital/Hospice in England and Wales [14]). If the hospital is located close to the place of residence, one might reasonably suppose that a patients' exposure to outdoor pollution might be reduced when confined indoors [15]. Epidemiological observations have shown that deaths associated with air pollution, specifically TSP and Particle Matter with a diameter less than $10 \mu \mathrm{m}\left(\mathrm{PM}_{10}\right)$, are disproportionately increased outside of hospital $[16,17]$. In addition, Jansen et al. 2002, found that the health effects of $\mathrm{PM}_{10}$ on cardiovascular disease and COPD in 14 U.S. cities decreased significantly as the proportion of homes with air conditioning increased [18]. Previous attempts at comparing risks in and out of hospital, such as Téllez-Rojo et al. 2001 and Zeka et al. 2006, have shown significant increased risk of death from respiratory or cardiovascular causes, in some cases up to a threefold increase. These studies have primarily used location at time of death without confirming location during exposure $[19,20]$. Failure to take account of hospitalisation during exposure could lead to further effect underestimation if a substantial fraction of the population experience reduced exposure in air-conditioned hospitals. A large proportion of subjects hospitalised during exposure might explain why some observational epidemiology studies based on routinely collected data may struggle to replicate previously demonstrated associations between pollution and pneumonia caused mortality [21].

Pneumonia occurs usually as a result of bacterial or viral infection. Often progressing rapidly within 24 hours, it presents symptoms such as coughing, chest pain, shortness of breath, and fever. Pneumonia can generally be diagnosed reliably through medical consultation and a chest radiography [22]. The relatively quick onset of the disease, short diagnosis period, and the time varying nature of air pollution exposure satisfies the conditions for employing the case-crossover design [23]. This design offers protection against possible subject level confounders without the need for complex modelling of mortality levels over time.

This study investigated the effect of 'black smoke' (BS) over 30 days prior to pneumonia mortality using a time-stratified case-crossover design [24]. We tested the primary hypothesis that estimated association would be greater in subjects who spent the exposure period in the 'community' (i.e. not in hospital) compared to those who spent some or all of the period in hospital. Members of the former group will be defined as subjects with a Community Death from Pneumonia (CDPs) which should be distinguished from CAP; CAP refers to a clinical category based on the source of pneumonia, CDP are a subgroup of CAP. Concurrently, hypothesis generating analyses explored how exposure affects mortality from pneumonia across the lag period. Subsequently, analyses based on the lag periods which showed effects on mortality were repeated for subgroups defined by gender and age. It is conceivable that differences in lifestyle between these groups could influence exposure or that there is increased susceptibility in older age, leading to differential observed effects of BS on CDPs.

\section{Methods}

Deaths due to pneumonia (ICD-9 codes 480-487 pre 2000) between January 1981 and December 1996 from Edinburgh, Scotland formed the cases. Separately, information was provided on all admissions to hospital caused by respiratory, cardiovascular, lung cancer, diabetes, and digestive related causes for the same time period and location. The Information Services Division of NHS National Services in Scotland provided both datasets, they included; date of death, age, gender, admission dates, primary and secondary cause of admission and death, and if the patient died in hospital. The two files - one of deaths and one of hospital admissions - were linked to determine if the subject had been in hospital during the 30 days prior to death. Community Acquired Pneumonia refers to those subjects who did not acquire pneumonia from a hospital. Subjects with CAP may subsequently enter and later die in hospital. These subjects will not be included in our data as they will have had at least one day in hospital altering their exposure and increasing their chances of a secondary Hospital Acquired Pneumonia infection. A death was considered to be a Community Death from Pneumonia if the hospital admission data showed that the subject had not been in 
hospital for any of the 30 days prior to death. A CDP subject is therefore a special subgroup of the CAP deaths.

Daily black smoke air pollution data was obtained from one centrally located background monitoring site and hourly ambient air temperature (between 7 am-11 pm) was provided by the Scottish Meteorological Office. This was used to give daytime mean temperature and mean pollution levels for the area. For each date of death, or case day, 'black smoke' daily results for the month prior were averaged firstly across $1-30$ days and then separately for 1-6, 7-12, 13-18, 19-24, and 25-30 days. These formed the exposure variables for the cases. Same day exposure (lag 0) was not included as pneumonia has a minimum $24 \mathrm{hr}$ incubation period [22], and same day exposure potentially includes exposure after death. Temperature displays temporal associations with pollution levels, and has previously been shown in this population to have an approximately double linear relationship with mortality, with a knot at $11^{\circ} \mathrm{C}$ [25]. Hence, two continuous temperature variables were calculated as, "high" ( $\mathrm{t}-11$ if $\geq 11^{\circ} \mathrm{C}, 0$ otherwise) and "low" ( $\mathrm{t}-11$ if $\leq 11^{\circ} \mathrm{C}, 0$ otherwise), where $\mathrm{t}$ is the daytime mean temperature. Average temperature across lags 1-30 days for both "low" and "high" variables was included in all models. Including pollution and temperature exposure lags (up to 30 days prior) reduces the chances of underestimating the exposure effect [11]. Further information regarding the data source and variable manipulations can be found elsewhere [25].

The 30 days prior to the date of death from pneumonia defined the case exposure period. In case-crossover designs, reduced bias allows for a time-stratified design to select matched control periods [26]. Control days were defined as all equivalent days of the week within the same month as the case day, in order to account for any weather, seasonal, or day of the week confounding [27]. For example, if the event occurred on the second Monday of May then the control days became all other Mondays of May. The control exposure period was then 30 days prior to the control day, and temperature and pollution variables were formed from these periods. Ethical approval for the study was granted by the NHS Research Ethics Committee (Ref - 11/NW/0768) and the Privacy Advisory Committee associated with the Information Services Division Scotland once appropriate measures had been implemented to ensure any participant information was securely stored.

\section{Statistical analysis}

Conditional logistic regression [28], initially compared average exposure between the case and controls over 30 days prior to death (Model 1 ). To investigate exposure during the 30 days, a lag-stratified model fitted five exposure variables each representing a 6 day lag period (Model 2). To avoid problems comparing estimates based on different period lengths (e.g. 30 days and 6 days) [25,29], all estimated coefficient were divided by the number of days on which the mean was based. Hence, BS results are expressed as percentage increase in risk associated with an increase of $10 \mu \mathrm{gm}^{-3}\left(\left(\mathrm{e}^{\mathrm{B}^{*} 10}-1\right)^{*} 100\right.$, where $\mathrm{B}=$ model coefficient) on any individual day within the lag period. Similarly, both continuous temperature variables are also expressed as a percentage increase in risk corresponding to a decrease in $1^{\circ} \mathrm{C}$ of temperature for any individual day during lag period $\left(\left(\mathrm{e}^{-\mathrm{B}}-1\right)^{*} 100\right)$. The association between BS and All Pneumonia deaths (AP) was estimated before restricting to the subgroup (CDP) deemed to have the greatest potential exposure. To test if a significant difference in exposure effects occurred, an interaction term was included to compare CDP and non-CDP for each BS lag term, and the Log-likelihood-ratio was used to test the difference.

Within each lag period the average daily value across the lag period was calculated so long as a minimum of four out of every six days contained a pollution estimate. The analysis was then performed with any subject with complete data for the case day and at least one control day. This resulted in 4.5\% and 4.3\% (AP and CDP, respectively) of subjects dropped due to either missing pollution data in the case day or all control days.

The effect of a change in exposure on an individual day might be expected to vary across subsequent days and eventually fall to zero [30]. An estimated effect might even become negative afterwards if the 'high risk' pool of subjects is depleted without sufficient replenishment, causing a mortality rate lower than the underlying rate [17]. In addition to lag-stratified, a quadratic distributed lag [30] estimated the lag time, L, before which the estimated effects are positive. For simplicity in further analysis, average exposure across the period $(0, \mathrm{~L})$ days was modelled for each gender and two age groups $(\leq 80$ and $>80)$. Analysis was performed using STATA version 11 [31].

\section{Results}

A total of 15,784 people had pneumonia as primary or secondary cause of death in Edinburgh between 1981 and 1996. However, missing pollution data (9.8\% of days during the time period) for either a subject's case day or all of the subject's control days meant only 14,346 cases with 47,431 control days were eligible for the analysis. Table 1 gives descriptive statistics for the daily average BS air pollution, air temperature and demographic characteristics for both all pneumonia (AP) and CDP (52.5\% of the AP) subject groups. Further, summary statistics indicated an inter-quartile range approximately $10 \mathrm{\mu gm}^{-3}$ for each lag period.

The percentage change in relative risk (\%RR), with 95\% confidence intervals, are given in Table 2 for an increase in $\mathrm{BS}$ of $10 \mathrm{\mu gm}^{-3}$ or a temperature decrease of $1^{\circ} \mathrm{C}$ on any 
Table 1 Descriptive statistics of exposure data and study subjects split by all pneumonia and community deaths from pneumonia

\begin{tabular}{|c|c|c|c|c|c|c|}
\hline & Mean & S.D. & Median & IQR & Min & Max \\
\hline Daily Ave Air Temp $\left({ }^{\circ} \mathrm{C}\right)$ & 9.4 & 5.1 & 9.4 & 8 & -12.7 & 24.48 \\
\hline Daily Ave BS $\left(\mu \mathrm{gm}^{-3}\right)$ & 12.7 & 13.3 & 9 & 10 & 1 & 194 \\
\hline Lag 1-6 days Ave BS $\left({\left.\mu \mathrm{gm}^{-3}\right)}^{-}\right.$ & 12.9 & 10.8 & 9.3 & 9.5 & 1 & 95.2 \\
\hline Lag 7-12 days Ave BS $\left({\left.\mu \mathrm{gm}^{-3}\right)}^{-}\right.$ & 13 & 11.1 & 9.5 & 9.6 & 1 & 95.2 \\
\hline Lag 13-18 days Ave BS $\left({\left.\mu \mathrm{gm}^{-3}\right)}^{-}\right.$ & 13.1 & 11.3 & 9.5 & 9.7 & 1 & 95.2 \\
\hline Lag 19-24 days Ave BS $\left({\left.\mu \mathrm{gm}^{-3}\right)}\right.$ & 13.1 & 11.2 & 9.5 & 9.5 & 1 & 95.2 \\
\hline 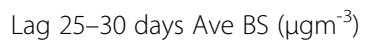 & 13 & 11.2 & 9.5 & 9.3 & 1 & 95.2 \\
\hline Lag 1-30 days Ave BS $\left({\left.\mu \mathrm{gm}^{-3}\right)}\right.$ & 13.2 & 9.2 & 10.1 & 9.3 & 2.7 & 73.2 \\
\hline Age (CDP) & 79.13 & 12.6 & 82 & 12 & 0 & 108 \\
\hline Age (Non-CDP) & 79.21 & 11.92 & 81 & 13 & 0 & 108 \\
\hline \multirow[t]{2}{*}{ Age (AP) } & 79.15 & 12.2 & 81 & 13 & 0 & 108 \\
\hline & \multicolumn{2}{|c|}{ Gender } & \multicolumn{3}{|c|}{ Age Grouped } & \\
\hline Categories & Male & Female & $<80$ & \multicolumn{2}{|c|}{$\geq 80$} & Total \\
\hline CDP only Subjects (\%) & $3,409(45.2)$ & $4,127(54.8)$ & $3,064(40.7)$ & \multicolumn{2}{|c|}{$4,472(59.3)$} & $7,536(100)$ \\
\hline Non-CDP only Subjects (\%) & $3,166(48.2)$ & $3,644(46.9)$ & $3,109(50.4)$ & \multicolumn{2}{|c|}{$3,701(45.3)$} & $6,810(47.5)$ \\
\hline All Pneumonia Subjects (\%) & $6,575(45.8)$ & $7,771(54.2)$ & $6,173(43.0)$ & \multicolumn{2}{|c|}{$8,173(57.0)$} & $14,346(100)$ \\
\hline
\end{tabular}

BS = Black Smoke.

individual day. MODEL 1 and 2 refers to the model with BS and temperature averaged over 1-30 days and BS split into five smaller lag periods, respectively. Correlation coefficients indicate strong correlation between average exposures in adjacent lag periods $(\approx 0.7)$ however the corresponding variance inflation factors $(\mathrm{VIF}=2.01$ to 2.78) did not indicate the presence of collinearity between the five lag periods in model 2 [32]. To easily compare effect sizes between lag periods the percentage change in relative risk corresponds to the effect of a change in BS or temperature on any individual day within the associated lag period. The differences in \%RR between AP and CDP along with corresponding significance levels are also given.

MODEL 1 considers the effects of exposure on each of

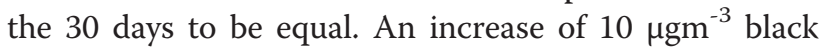

Table 2 Percent change in risk for lagged black smoke air pollution and pneumonia mortality: repeated for AP (All pneumonia), CDP (Community death from pneumonia) \& Non-CDP

\begin{tabular}{|c|c|c|c|c|c|c|c|c|c|c|}
\hline & \multirow[b]{2}{*}{ Lag (days) } & \multicolumn{2}{|r|}{ AP } & \multicolumn{2}{|c|}{ CDP only } & \multicolumn{2}{|c|}{ Non - CDP only } & \multirow[b]{2}{*}{$\underset{\substack{|C D P|-|A P| \\
\text { diff }}}{|c|}$} & \multirow[b]{2}{*}{$\begin{array}{l}\mid C D P-N o n \\
\text { CDP| diff }\end{array}$} & \multirow[b]{2}{*}{ P-va } \\
\hline & & $\begin{array}{c}\% \text { RR } \\
\text { change }\end{array}$ & 95\% C.I. & $\begin{array}{c}\% \text { RR } \\
\text { change }\end{array}$ & 95\% C.I. & $\begin{array}{c}\% \text { RR } \\
\text { change }\end{array}$ & 95\% C.I. & & & \\
\hline $\begin{array}{l}\text { MODEL } 1 \text { Black } \\
\text { Smoke }\end{array}$ & $1-30$ & $0.08 \%$ & $-0.17 \%, 0.35 \%$ & $0.19 \%$ & $-0.16 \%, 0.58 \%$ & $0.01 \%$ & $-0.34 \%, 0.40 \%$ & $0.11 \%$ & $0.18 \%$ & 0.285 \\
\hline Air Temp "Low" & $1-30$ & $0.20 \%$ & $0.11 \%, 0.29 \%$ & $0.22 \%$ & $0.09 \%, 0.35 \%$ & $0.16 \%$ & $0.03 \%, 0.30 \%$ & $0.02 \%$ & $0.06 \%$ & 0.502 \\
\hline Air Temp "High" & $1-30$ & $-0.05 \%$ & $-0.20 \%, 0.10 \%$ & $-0.20 \%$ & $-0.40 \%, 0.00 \%$ & $0.11 \%$ & $-0.11 \%, 0.34 \%$ & $0.15 \%$ & $0.31 \%$ & 0.059 \\
\hline \multirow{5}{*}{$\begin{array}{l}\text { MODEL } 2 \text { Black } \\
\text { Smoke }\end{array}$} & $1-6$ & $0.12 \%$ & $-0.37 \%, 0.62 \%$ & $0.56 \%$ & $-0.14 \%, 1.29 \%$ & $-0.31 \%$ & $-0.99 \%, 0.41 \%$ & $0.44 \%$ & $0.87 \%$ & 0.022 \\
\hline & $7-12$ & $0.05 \%$ & $-0.42 \%, 0.53 \%$ & $0.32 \%$ & $-0.33 \%, 1.00 \%$ & $-0.25 \%$ & $-0.92 \%, 0.45 \%$ & $0.28 \%$ & $0.57 \%$ & 0.023 \\
\hline & $13-18$ & $0.40 \%$ & $-0.08 \%, 0.90 \%$ & $0.71 \%$ & $0.03 \%, 1.42 \%$ & $0.14 \%$ & $-0.56 \%, 0.86 \%$ & $0.31 \%$ & $0.57 \%$ & 0.163 \\
\hline & $19-24$ & $-0.09 \%$ & $-0.55 \%, 0.38 \%$ & $-0.16 \%$ & $-0.79 \%, 0.50 \%$ & $0.05 \%$ & $-0.63 \%, 0.75 \%$ & $0.06 \%$ & $0.21 \%$ & 0.272 \\
\hline & $25-30$ & $-0.11 \%$ & $-0.57 \%, 0.36 \%$ & $-0.39 \%$ & $-1.01 \%, 0.25 \%$ & $0.34 \%$ & $-0.35 \%, 1.06 \%$ & $0.28 \%$ & $0.73 \%$ & 0.008 \\
\hline Air Temp "Low" & $1-30$ & $0.19 \%$ & $0.10 \%, 0.29 \%$ & $0.19 \%$ & $0.06 \%, 0.32 \%$ & $0.18 \%$ & $0.05 \%, 0.32 \%$ & $0.00 \%$ & $0.01 \%$ & 0.487 \\
\hline Air Temp "High" & $1-30$ & $-0.05 \%$ & $-0.19 \%, 0.10 \%$ & $-0.20 \%$ & $-0.39 \%, 0.01 \%$ & $0.09 \%$ & $-0.12 \%, 0.33 \%$ & $0.15 \%$ & $0.29 \%$ & 0.058 \\
\hline
\end{tabular}

\%RR Change - percentage change in Relative Risk, associated with an increase of $10 \mu \mathrm{gm}^{-3} \mathrm{BS}$ or a decrease of $1^{\circ} \mathrm{C}$, on any individual day within the lag period, with corresponding $95 \%$ Confidence Interval (95\% C.I.).

Model 1 - One 30 day lag, Model 2 - The 30 days split into 5 lags of 6 days each fitted simultaneously.

CDP - AP Diff - The difference in the magnitude of the effect size between AP and CDP (CDP-AP).

CDP-Non CDP Diff - The difference in the effect size between CDP and Non-CDP (CDP - Non CDP). 
smoke on any of the 30 days, showed a small rise in AP relative risk increasing to $0.19 \%$ in the CDP group, resulting from a \%RR difference of $-0.18 \%$ between CDP and non-CDP subjects (CDP-non-CDP \%RR). When the 30 days is split into 5 lag periods (MODEL 2), the magnitude of the effect is always larger in the CDP subjects, of whom the largest changes in \%RR are seen in the 16, 7-12, and 13-18 day lags. This 18 day period prior to death appeared to be the high risk period, as an increase $\% R R$ is observed in $1-6,7-12$, and $13-18$ day lags whereas a decrease is observed in the 19-24 and 2530 day lags. Figure 1 plots the change in log rate ratio associated with the 30 day lag period for both AP and $\mathrm{CDP}$ as modelled using the quadratic lag distribution model. As suggested in Table 2, the CDP group showed larger effects with a more rapid decline crossing zero at approximately 21 days, almost 2 days earlier than the more gradual AP decline in risk.

Low Temperature effects changed very little, a $1^{\circ} \mathrm{C}$ decrease corresponded to an increase in relative risk approximately the same for both AP and CDP. In comparison, a $1^{\circ} \mathrm{C}$ decrease in high temperature shows a small decrease in risk in AP that increases in magnitude in the CDP group.

A secondary analysis concentrated on the minimum lag period where increased risk was observed (18 days) and split the data into the subgroups; gender (male/ female), and age $(<80 / \geq 80)$. In all but low temperature for males \& age $<80$ an increase in \%RR was seen in the CDP group. The CDP subjects indicated a stronger \%RR in males $(0.83 \%$ - $95 \%$ C.I. $0.21 \%, 1.51 \%)$ compared to females $(0.61 \%-95 \%$ C.I. $0.08 \%, 1.19 \%)$. Subjects aged 80 or above showed larger relative risk in AP $(0.37 \%$ - $95 \%$ C.I. $0.00 \%, 0.77 \%$; to $0.23 \%$ - $95 \%$ C.I. $-0.17 \%, 0.66 \%$ in $<80$ ) but the difference disappeared when restricted to CDP subjects $(0.71 \%-95 \%$ C.I. $0.18 \%, 1.30 \%$ and $0.71 \%-95 \%$ C.I. $0.10 \%, 1.38 \%$, respectively). Analysis was repeated for

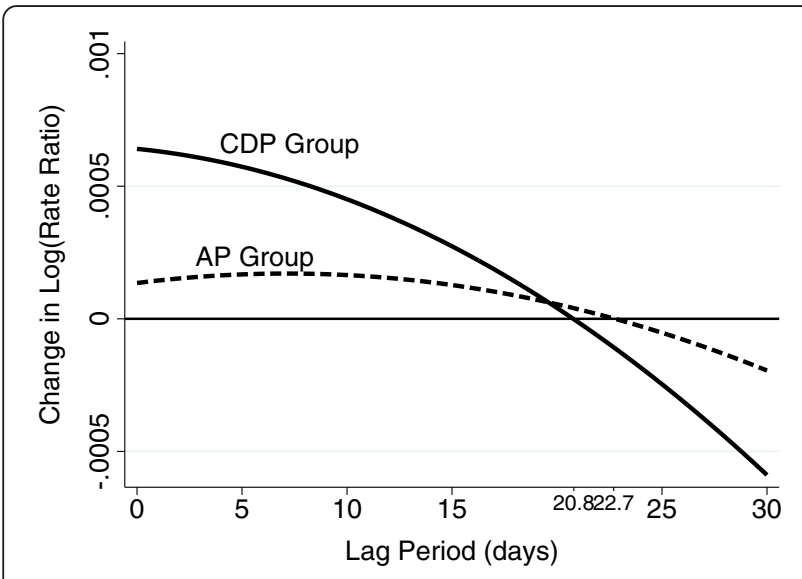

Figure 1 The quadratic lag distribution model for subjects with all pneumonia and community deaths from pneumonia. subjects aged less than 65 to investigate different age distributions of pneumonia subtypes. Even though the effect sizes showed slightly larger differences in magnitude compared to the less than 80 group they were highly imprecise due to substantially smaller sample size, hence they have not been reported here. Comparisons using the loglikelihood test were performed between the gender and age groups; in all cases the difference was non-significant at the $5 \%$ level. Further information can be found in the Additional file 1: Table S1.

\section{Discussion}

These findings suggest that a subject's location is an important consideration when assessing the effect of ambient air pollution on pneumonia mortality. In this study, the percentage relative risk of air pollution was significantly higher in CDP compared to AP by $0.44 \%$ (1-6 day lag), $0.28 \%$ (7-12 day lag) with a further important but non-significant increase of $0.31 \%$ in the 13-18 day lag. The 18 days prior to death indicated a lag period of increased risk, with the largest relative risk $(\mathrm{CDP}=0.71 \%(0.03 \%, 1.42 \%))$ in the $13-18$ day lag period. Prior to the $18^{\text {th }}$ day a decrease in relative risk occurred, with the magnitude increasing in the lag period furthest from the event. The quadratic distributed lag plot confirmed an increased risk period of 20-22 days prior to the event. The decrease effect on mortality may be due to a small pool of people susceptible to dying from pollution effects [17]. Initial changes in pollution cause the pool to diminish more rapidly than it can be replenished, creating a net deficit in the number of susceptible subjects; this leaves a larger proportion of stronger subjects to experience the longer lag periods and hence, a reduction in deaths.

In all lag periods, irrespective of the direction, the magnitude of the effect is greater in the CDP group. Hence, significant associations between BS and pneumonia mortality may have been overlooked in previous studies that have not taken into account location during exposure. Removing those who may have had a misleading exposure (due to hospitalisation) may have given a larger observed effect. Increased risk may be experienced in community based subjects due to reduced accesses to medical care that may have been able to prevent early symptoms from progressing to a critical phase. The bias associated with earlier recognition, and more timely and aggressive therapeutic intervention may be the reason for the apparently negative \%RR associated with an increase in BS of $10 \mu \mathrm{gm}^{-3}$ within the "non-CDP" only subject group.

This study finds a similar increase in BS exposure effects when compared to U.S. studies of TSP/PM 10 on all cause mortality when restricted to deaths located outside of hospital $[16,17]$. Black smoke contains finer particle fractions dominated by combustion emissions 
which are more closely associated with health outcomes than $\mathrm{PM}_{10}$ or $\mathrm{PM}_{2.5}$, and so BS filter darkness measurements are a better marker for harmful combustion-related particles [33]. Currently, few published articles comparing indoor and outdoor BS levels exist. Gotschi et al. compared indoor and outdoor BS and $\mathrm{PM}_{2.5}$ for 186 homes in Athens, Basel, Helsinki and Prague. The median indoor-outdoor ratios of BS were slightly less than $\mathrm{PM}_{2.5}$, however, Spearman correlation coefficients were larger, possibly due to stronger indoor influences on $\mathrm{PM}_{2.5}$ [34]. Hoek et al. gave filter darkness regressions slopes (0.63-0.84) between indoor-outdoor concentrations in homes of four European cities [35]. Limited information with inconsistent outcomes, often due to small sample sizes $(\mathrm{N} \leq 50)$, is available comparing indoor and outdoor personal particulate matter exposure [36-38]. Janssen N.A.H et al. investigated personal, indoor and outdoor fixed site exposure to $\mathrm{PM}_{10}$ in 37 participants from Amsterdam and $\mathrm{PM}_{2.5}$ and $\mathrm{BS}$ in 36 and 46 participants from Amsterdam and Helsinki respectively. Sampling was taken for $24 \mathrm{hr}$ periods, bi-weekly, over six months. In both cases, median concentrations were found in the personal monitors, followed by outdoor monitoring and then indoor. High correlations were produced between personal and outdoor fixed site monitors indicating that fixed site monitors are a good representation of the day-to-day variation in particulate matter exposure $[39,40]$. However, high correlation does not imply the same absolute levels. The underlying premise; that exposure to airborne pollutants is reduced in hospitalised subjects; is supported by Wang et al. which showed a reduction in indoor concentrations of $\mathrm{PM}_{10}$ and $\mathrm{PM}_{2.5}$ in 2 of 4 hospitals in Guangzhou, China [41]. Subsequently, Wang et al. and later Morawska et al. further determined that a mechanical ventilation air conditioning system produced the lowest indooroutdoor $\mathrm{PM}_{10}$ ratios [42,43]. Indoor air quality is an important issue for hospitals. However, currently the quantity of literature available on the relationship between indoor and outdoor air pollution, particularly regarding hospitals, is limited. Further observational studies are required to supplement understanding of the reduction and fluctuations in indoor hospital air pollution concentrations, in terms of distance from combustion sources, changes in ventilation systems and meteorological conditions [44].

One possible alternative explanation for the increase in relative risk occurring within the CDP group is that exposure may have a differing interaction with certain types of pneumonia that are specifically associated with the community. Pneumonia infection can be caused by a variety of micro-organisms. Hospital Acquired Pneumonia is primarily caused by Staphylococcus aureus or Gramnegative enterobacteria, and CAP is most commonly Streptococcus pneumoniae (35\% of CAP cases) [45].
Streptococcus pneumonia has an incubation period of 13 days, shorter than other pathogens such as Haemophilus influenzae and Mycoplasma pneumonia with incubation periods of 2-4, and 6-32 days respectively [22]. Variation in source and incubation period may be a contributing confounding factor to the difference in BS effect on CDP mortality. Limited information from ICD coding constructed from the death certificate, allowed the pneumonia deaths to be classified into; bronchopneumonia (81\%), pneumococcal \& streptococcal pneumonia (5\%), organism unspecified (13\%), and all others (1\%), of which $67 \%$, $52 \%, 47 \%$, and $35 \%$, respectively were CDP subjects. Change in exposure effects on differing underlying causes of pneumonia may be a possible explanation for higher relative risk in CDP deaths. If type of pneumonia was the only explanation for higher RR, then we might expect the RRs for BS within the categories of pneumonia to be the same. In fact, the same pattern of a higher RR for CDP compared to hospital deaths was found for; bronchopneumonia, and pneumococcal \& streptococcal types (although not for; organism unspecified and all other types which had much smaller sample sizes reducing the power available to determine the true effect).

However, pollution itself may be the causal factor. Particulate pollution may increase the risk of contracting pneumonia in a number of ways; by impairing microbial clearance via the mucociliary mechanism [46], hindering macrophage phagocytosis [47], or causing intense capillary engorgement and loss of epithelium [48]. These effects might require an indeterminate dose (product of exposure concentration, respiratory minute volume and time) to materialise before manifesting in an increased susceptibility to pneumonia mortality. The temporal relationship between air pollution and pneumonia death analysed here may therefore comprise a period of chemical insult before, as well as a diagnosis to death interval enveloping the 'incubation period' as classically defined. These varying and relatively indeterminate periods may thus explain why $\% R R$ is reduced in the 7-12 day lag compared to $1-6$ and 13-18 day lags (Table 2).

A comparison of the BS lag periods within the two models indicated the possible presence of mortality displacement within the data. Mortality displacement, also known as harvesting, is the accelerated progression of a frail sub-population to death followed by a delay in its replenishment. This is thought to be illustrated by an increase in the death rate from its baseline for a certain period after exposure, followed by a period when the death rate seems to be below expected [17]. In Table 2 the lags post 18 days seems to suggest a negative relative risk but in fact this may be due to the shrinking of the at risk population. When the overall effect across the 30 days is estimated (Table 1), the positive and negative estimates balance out to some extent. These results are 
therefore consistent with the mortality displacement phenomenon. Pneumonia mortality may be more susceptible to 'harvesting' as pneumonia is prevalent in the elderly $[9,49]$ and is often the final cause in the chain of causes leading to death, implying a high incidence in the frail sub-population compared to the general population. If mortality displacement is present then it would have been easy to miss any risk period if the model only included one term representing average black smoke across 30 days.

The stronger risk experienced for the 18 days prior to death in males within the CDP group may be due to a more outdoors lifestyle in males causing an increased interaction with exposure. Younger subjects may also be expected to experience higher exposure to outdoor pollution concentrations. Yet we found no age group difference in risk within the CDP group possibly due to relatively few subjects aged 65 or less $(9.2 \%)$, or due to elderly patients being allowed to die at home rather than in hospital. One could further argue that it is difficult to accurately determine the exposure level for these subjects as the area contains only one exposure measurement site. Any exposure misclassification could be reduced if the number of measurement sites could be increased, making it easier to evaluate local variations in pollution levels. Even then, it is difficult to determine a subject location during the entire exposure period, especially when multiple control exposure periods are used. Other than to explore the relationship between BS and mortality; restricting the data to an 18 day lag period was not a part of our original aims and so we do recognise that we lose some validity in our p-values. However, as the 18 day lag period showed significant increases in risk we felt it was worth investigating further.

The study time period (1981-1996) does not necessarily limit these results to a historical interest only given that the primary aim was to compare the effect of pollution on subjects in the community versus within hospital. In fact, the higher outdoor concentrations of black smoke in the 1980 's and early 1990's were advantageous for testing this hypothesis given that higher pollution concentrations meant that the incidence of pollution related pneumonia mortality would have been higher and this would make the difference in risk, if any, associated with indoor and outdoor exposure easier to detect. The dominant source of black smoke during both the study period and present day were from road vehicles as most smoke control procedures to reduce combustion of coal for domestic heating and industrial energy would already have been implemented by the start of the study period. However, the chemical composition of fine black particles may have altered somewhat since the study period as a result of technological changes in vehicle engine and emissions control systems. It is not possible to directly characterise the extent of such changes as it is not possible to selectively collect black particles from non-black particles during atmospheric sampling for chemical analyses.

\section{Conclusions}

In conclusion, evidence suggests that a subject's location is an important factor in relation to their likelihood of pneumonia mortality due to particulate pollution exposure. Including subjects who may have a lower exposure may increase bias in your results and as shown here underestimate the true effect of exposure on pneumonia deaths. The risk to mortality in all subjects, and in particular within the CDP group, tends to last a minimum of 18 days and peaks at the 13-18 day lag. This confirms that air pollution effects do exist beyond short term exposure periods such as 1-3 days, making it is important to investigate extended exposure periods of at least two to three weeks prior to death.

\section{Additional file}

Additional file 1: Table S1. Subgroup analysis of 18 day exposure split by subject characteristics; split by AP, CDP \& Non-CDP.

\section{Abbreviations}

AP: All pneumonia; BS: Black smoke; CAP: Community acquired pneumonia; CDP: Community deaths from pneumonia; COPD: Chronic obstructed pulmonary disease; PM10: Particle matter with an aerodynamic diameter less than 10 .gm $^{-3}$; PM2.5: Particle matter with an aerodynamic diameter less than $2.5 \mathrm{\mu gm}^{-3}$; \%RR: Percentage relative risk; TSP: Total suspended particles; VIF: Variance inflation factor.

\section{Competing interests}

The authors declare that they have no competing interests.

\section{Authors' contributions}

MG performed the statistical analysis and drafted the paper. RM contributed to study idea, its design, statistical analysis and provided reviewers comments. MC helped conceive the study, provided advice, and reviewed the paper. IB contributed to the discussion and reviewed the paper. RA conceived of the study, participated in acquiring the data, provided advice for the discussions and reviewed the paper. All authors read and approved the final manuscript.

\section{Acknowledgments}

This work was supported by a research grant, number G9900747, from the Medical Research Council to RMA and colleagues. The views expressed in this publication are those of the authors and not necessarily those of the Medical Research Council. We thank Mr James Boyd and colleagues in the Information and Services Division of the National Health Service in Scotland for the provision of hospitalisation and mortality data.

\section{Author details}

${ }^{1}$ Biostatistics Group, University of Manchester, Jean McFarlane Building, Oxford Road, Manchester M13 9PL, England, UK. ${ }^{2}$ Centre for Occupational and Environmental Health, The University of Manchester, Humanities Building, Oxford Road, Manchester M13 9PL, England, UK. ${ }^{3}$ Department of Civil Engineering, University of Strathclyde, John Anderson Building, 107 Rottenrow, Glasgow G4 ONG, Scotland, UK. 


\section{References}

1. Anderson HR, de Leon AP, Bland JM, Bower JS, Strachan DP: Air pollution and daily mortality in London: 1987-92. Br Med J 1996, 312:665-669.

2. Samet JM, Dominici F, Curriero FC, Coursac I, Zeger SL: Fine particulate air pollution and mortality in 20 US Cities, 1987-1994. N Engl J Med 2000, 343:1742-1749.

3. Goldberg MS, Burnett RT, Bailar JC, Brook J, Bonvalot Y, Tamblyn R, Singh R, Valois MF, Vincent R: The association between daily mortality and ambient air particle pollution in Montreal, Quebec 2. Cause-specific mortality. Environ Res 2001, 86:26-36.

4. Schwartz J: Particulate Air-pollution and chronic respiratory-disease. Environ Res 1993, 62:7-13.

5. Zanobetti A, Bind MAC, Schwartz J: Particulate air pollution and survival in a COPD cohort. Environmental: Health; 2008:7.

6. ONS: Deaths Registered in England and Wales in 2011, by Cause. 2012 http://www.ons.gov.uk/ons/dcp171778_284566.pdf.

7. Schwartz J, Dockery DW: Increased mortality in Philadelphia associated with daily Air-pollution concentrations. Am Rev Respir Dis 1992, 145:600-604.

8. Halonen JI, Lanki T, Yli-Tuomi T, Tiittanen P, Kulmala M, Pekkanen J: Particulate air pollution and acute cardiorespiratory hospital admissions and mortality among the elderly. Epidemiology 2009, 20:143-153.

9. Zanobetti A, Woodhead M: Air pollution and pneumonia the "old man" has a new "friend". Am J Respir Crit Care Med 2010, 181:5-6.

10. Neupane B, Jerrett M, Burnett RT, Marrie T, Arain A, Loeb M: Long-term exposure to ambient Air pollution and risk of hospitalization with community-acquired pneumonia in older adults. Am J Respir Crit Care Med 2010, 181:47-53.

11. Goodman PG, Dockery DW, Clancy L: Cause-specific mortality and the extended effects of particulate pollution and temperature exposure. Environ Health Perspect 2004, 112:179-185.

12. Zeka A, Zanobetti A, Schwartz J: Short term effects of particulate matter on cause specific mortality: effects of lags and modification by city characteristics. Occup Environ Med 2005, 62:718-725.

13. Maynard D, Coull BA, Gryparis A, Schwartz J: Mortality risk associated with short-term exposure to traffic particles and sulfates. Environ Health Perspect 2007, 115:751-755.

14. Office for National Statistics: Mortality Statistics: Deaths registered in 2008. 2009. http://www.ons.gov.uk/ons/rel/vsob1/mortality-statistics-deathsregistered-in-england-and-wales-series-dr-/2008/mortality-statistics-deathsregistered-series-dr-pdf.

15. Byrne M: Aerosols exposed. Chem Br 1998, 34:23-26.

16. Schwartz J: What Are people dying of on high Air-pollution days. Environ Res 1994, 64:26-35.

17. Schwartz J: Is there harvesting in the association of airborne particles with daily deaths and hospital admissions? Epidemiology 2001, 12:55-61.

18. Janssen NAH, Schwartz J, Zanobetti A, Suh HH: Air conditioning and source-specific particles as modifiers of the effect of PM10 on hospital admissions for heart and lung disease. Environ Health Perspect 2002, 110:43-49.

19. Tellez-Rojo MM, Romieu I, Ruiz-Velasco S, Lezana MA, Hernandez-Avila MM: Daily respiratory mortality and PM10 pollution in Mexico City. Eur Respir J 2001, 18:1076.

20. Zeka A, Zanobetti A, Schwartz J: Individual-level modifiers of the effects of particulate matter on daily mortality. Am J Epidemiol 2006, 163:849-859.

21. Gouveia N, Fletcher T: Respiratory diseases in children and outdoor air pollution in Sao Paulo, Brazil: a time series analysis. Occup Environ Med 2000, 57:477-483.

22. Hawker J: Communicable disease control handbook. Malden, Mass. Oxford: Blackwell Pub; 2005.

23. Rothman KJ, Greenland S: Modern epidemiology. Philadelphia: LippincottRaven; 1998.

24. Jaakkola JJ: Case-crossover design in air pollution epidemiology. Eur Respir J Suppl 2003, 40:81s-85s

25. Carder M, McNamee R, Beverland I, Elton R, Cohen GR, Boyd J, Agius RM: The lagged effect of cold temperature and wind chill on cardiorespiratory mortality in Scotland. Occup Environ Med 2005, 62:702-710.

26. Lumley T, Levy D: Bias in the case-crossover design: implications for studies of air pollution. Environmetrics 2000, 11:689-704.

27. Janes H, Sheppard L, Lumley T: Overlap bias in the case-crossover design, with application to air pollution exposures. Stat Med 2005, 24:285-300.
28. Hosmer DW, Lemeshow S, Cook ED: Applied logistic regression. New York Chicheter: John Wiley \& Sons; 2000.

29. Beverland IJ, Carder M, Cohen GR, Heal MR, Agius RM: Associations between short/medium-term variations in black smoke air pollution and mortality in the Glasgow conurbation, UK. Environ Int 2012, 50:1-6.

30. Schwartz J: The distributed lag between air pollution and daily deaths. Epidemiology 2000, 11:320-326.

31. StataCorp LP: Getting started with Stata for Windows: release 11. College Station, Tex: StataCorp LP; 2009.

32. Menard SW: Applied logistic regression analysis. Thousand Oaks CA London: Sage Publications; 2002.

33. WHO: Health Aspects of Air Pollution with Particle Matter, Ozone and Nitrogen Dioxide. 2003. www.euro.who.int/document/e79097.pdf.

34. Gotschi T, Oglesby L, Mathys P, Monn C, Manalis N, Koistinen K, Jantunen M, Hanninen O, Polanska L, Kunzli N: Comparison of black smoke and PM2.5 levels in indoor and outdoor environments of four European cities. Environ Sci Technol 2002, 36:1191-1197.

35. Hoek G, Kos G, Harrison R, de Hartog J, Meliefste K, ten Brink H, Katsouyanni K, Karakatsani A, Lianou M, Kotronarou A, et al: Indoor-outdoor relationships of particle number and mass in four European cities. Atmos Environ 2008, 42:156-169.

36. Williams R, Creason J, Zweidinger R, Watts R, Sheldon L, Shy C: Indoor, outdoor, and personal exposure monitoring of particulate air pollution: the Baltimore elderly epidemiology-exposure pilot study. Atmos Environ 2000, 34:4193-4204.

37. Vallejo M, Lerma C, Infante O, Hermosillo AG, Riojas-Rodriquez H, Cardenas M: Personal exposure to particulate matter less than $2.5 \mathrm{mu}$ m in Mexico City: a pilot study. J Expo Anal Environ Epidemiol 2004, 14:323-329.

38. Brauer M, Hirtle RD, Hall AC, Yip TR: Monitoring personal fine particle exposure with a particle counter. J Expo Anal Environ Epidemiol 1999, 9:228-236.

39. Janssen NAH, Hoek G, Brunekreef B, Harssema H, Mensink I, Zuidhof A: Personal sampling of particles in adults: relation among personal, indoor, and outdoor air concentrations. Am J Epidemiol 1998, 147:537-547.

40. Janssen NAH, Lanki T, Hoek G, Vallius M, de Hartog JJ, Van Grieken R, Pekkanen J, Brunekreef B: Associations between ambient, personal, and indoor exposure to fine particulate matter constituents in Dutch and Finnish panels of cardiovascular patients. Occup Environ Med 2005, 62:868-877.

41. Wang XH, Bi XH, Chen DH, Sheng GY, Fu JM: Hospital indoor respirable particles and carbonaceous composition. Build Environ 2006, 41:992-1000.

42. Morawska L, Jamriska M, Guo H, Jayaratne ER, Cao M, Summerville S: Variation in indoor particle number and PM2.5 concentrations in a radio station surrounded by busy roads before and after an upgrade of the HVAC system. Build Environ 2009, 44:76-84.

43. Wang XH, Bi XH, Sheng GY, Fu HM: Hospital indoor PM10/PM2.5 and associated trace elements in Guangzhou, China. Sci Total Environ 2006, 366:124-135.

44. Shilton V, Giess P, Mitchell D, Williams C: The relationships between indoor and outdoor respirable particulate matter: Meteorology, chemistry and personal exposure. Indoor Built Environ 2002, 11:266-274.

45. Wilkinson M, Woodhead M: Pneumonia. Medicine 2004, 32:129-134.

46. Saldiva PHN, King M, Delmonte VLC, Macchione M, Parada MAC, Daliberto ML, Sakae RS, Criado PMP, Silveira PLP, Zin WA, et al: Respiratory alterations Due to urban Air-pollution - an experimental-study in rats. Environ Res 1992, 57:19-33.

47. Zhou HW, Kobzik L: Effect of concentrated ambient particles on macrophage phagocytosis and killing of Streptococcus pneumoniae. Am J Respir Cell Mol Biol 2007, 36:460-465.

48. Knox EG: Atmospheric pollutants and mortalities in English local authority areas. J Epidemiol Community Health 2008, 62:442-447.

49. Sousa D, Justo I, Dominguez A, Manzur A, Izquierdo C, Ruiz L, Nebot M, Bayas JM, Celorrio JM, Varona W, et al: Community-acquired pneumonia in immunocompromised older patients: incidence, causative organisms and outcome. Clin Microbiol Infect 2013, 19:187-192.

doi:10.1186/1476-069X-12-97

Cite this article as: Gittins et al:: Has the short-term effect of black smoke exposure on pneumonia mortality been underestimated because hospitalisation is ignored: findings from a case-crossover study.

Environmental Health 2013 12:97. 